\title{
EFECTO AGUDO DE UNA SESIÓN DE IMPROVISACIÓN TEATRAL Y DE FÚTBOL SALA EN EL ESTADO DE ÁNIMO DE ADOLESCENTES PRIVADOS DE LIBERTAD DEL CENTRO DE FORMACIÓN ZURQUÍ
}

\author{
Vivian Rodríguez Barquero, Gerardo Araya Vargas y Walter Salazar Rojas \\ Escuela de Educación Física y Deportes, Universidad de Costa Rica \\ E-mail: vivianrodriguez@costarricense.cr
}

\section{Resumen:}

Rodríguez-Barquero, V.; Araya-Vargas, G. y Salazar-Rojas, W. (2007). Efecto agudo de una sesión de improvisación teatral y de fútbol sala en el estado de ánimo de adolescentes privados de libertad del Centro de Formación Zurquí. Revista de Ciencias del Ejercicio y la Salud, 5(1), 47-54. El propósito del estudio fue comparar el efecto agudo de una sesión de improvisación teatral (actividad física recreativa) y el de una sesión de fútbol sala (actividad deportiva competitiva), sobre los estados de ánimos de varones adolescentes privados de libertad. Metodología: muestra de 21 sujetos privados de libertad indiciados y sentenciados con promedio de edad de 15,8 $\pm 1,79$ años y $16,125 \pm 0,81$ años respectivamente; realizaron una sesión de improvisación y una de fútbol sala, ambas de una hora. Las mediciones del estado de ánimo se realizaron por medio del perfil de estados de ánimo (POMS) pre y post de cada sesión. Resultados: se encontraron efectos agudos significativos y positivos en tensión, fatiga, depresión y cólera-ira, luego de la sesión de improvisación teatral, a diferencia de lo ocurrido con la sesión de deporte, en la cual se dieron conductas de enfrentamiento (motivadas por la competencia en sí) que alteraron el estado anímico de los participantes. Conclusiones: parece que la actividad físico recreativa beneficia más que el deporte, los estados de ánimo. Esta línea de estudio es prometedora y debería profundizarse más. Es importante la investigación del teatro y la expresión corporal, pues sus efectos terapéuticos, pueden ser introducidos como tratamientos de bajo costo.

PALABRAS CLAVES: Privados de libertad, adolescentes, improvisación teatral, fútbol, estado de ánimo.

\section{INTRODUCCIÓN}

\section{Beneficios psicológicos del ejercicio}

Se ha encontrado evidencia significativa en estudios experimentales sobre los beneficios psicológicos del ejercicio (Dubbert, 2002; O'neal, Dunn, Martinsen, 2000; Martinsen, 1993; Bidle, 1993; Carson y Crabbe, 2000; Tucker, 1990; Kirkcaldy, 1990), como tratamiento alternativo para la depresión, ansiedad, estado de ánimo, estrés, etc.

Aspectos psicológicos de la actividad física, como el contacto social o la percepción de maestría en el ejercicio, pueden contribuir a estos efectos beneficiosos. Otros de los efectos positivos de la actividad física son: un aumento de la percepción de salud, de la sensación de auto-suficiencia, de la imagen corporal, auto-concepto y un mayor manejo de estrés (Dubbert, 2002). En general, continuando con Dubbert, se ha encontrado una respuesta somato-terapéutica, es decir, que los cambios en el cuerpo influencian la actitud mental, induciendo a estados psicológicos positivos.

Se necesitan estudios más conclusivos acerca de estos efectos, para determinar con más exactitud el alcance terapéutico del ejercicio, ya que faltan más instrumentos de evaluación psicológica confiables, pero a pesar de estas limitaciones, ahora es posible confirmar los efectos beneficiosos en diferentes poblaciones.

\section{Adolescentes privados de libertad y la delincuencia}

En Estados Unidos, corrientes de investigación en delincuencia y técnicas clínicas, buscan dar tratamiento a 
adolescentes privados de libertad, ya que entre 1989 y 1997 , se incrementó un $22 \%$ los arrestos a menores de edad, un 2,3\% de las causas de arresto son violentas. Por otra parte, el costo de un adolescente envuelto en drogas o crimen es de millones de dólares para el gobierno. Estos adolescentes están expuestos a violencia física y sexual, uso de drogas, armas, negligencia física y emocional, lo cual desencadena en un comportamiento de emociones volátiles, resistencia al tratamiento y múltiples desordenes psicológicos. Un estudio realizado con 120 sujetos determinó que el $23 \%$ sufrían de desordenes afectivos, el principal era la depresión, de acuerdo con Rutherford (1989), mencionado por Granello y Hanna (2003).

Hay pocos estudios nacionales en el campo de la actividad física y privados de libertad, de hecho, el único encontrado demuestra una disminución significativa en la ansiedad estado y rasgo de esta población gracias a la actividad física, como una herramienta útil para la reinserción de los privados de libertad a la sociedad. (Ureña, Mora, Godinez, Rodríguez, 2004). Además Calderón, Delgado y Oviedo (2005), en un estudio hecho con privados de libertad encontraron efectos (agudo y crónico) positivos en el estado de ánimo, por medio de dos programas: uno recreativo-deportivo y otro deportivo-competitivo. Por su parte, Vargas en 1995, realizó un estudio con drogadictos, mostrando una mejoría significativa, física, y una mejoría leve en autoconcepto, autoestima, gracias al ejercicio.

Se han realizado estudios de los patrones psicológicos de estos adolescentes encontrando una tendencia a la depresión, baja autoestima, optimismo exagerado y percepción de la soledad, más elevada que adolescentes en libertad (Kunzi 1999). Además otros estudios clínicos (Allen, Marsh, McFarland, McElhaney y Land 2002; Sussman, Jones, Wilson y Kann, 2002), con esta población, reconocen el rol de la adaptación al medio, sin temor o inseguridad, como una pieza importante en la tendencia a la delincuencia, violencia o uso de drogas.

Forrest, Tambor, Riley, Ensminger y Starfield en el 2000 realizaron un estudio comparativo sobre el perfil de salud entre adolescentes privados de libertad $y$ colegiales, los resultados mostraron el Peor Perfil de salud en los adolescentes privados de libertad con $69,8 \%$ contra $37,3 \%$ de los colegiales, además el perfil de salud excelente de los adolescentes privados de libertad es de $6,4 \%$ contra un $34,2 \%$ en colegiales.

Esto demuestra una diferencia abismal entre estas dos poblaciones y llama la atención para observar la necesidad de programas de rehabilitación para conseguir un nivel básico de salud que les permita modificar sus conductas de riesgo social. Los programas, deberían de incluir dinámicas para mejorar sus habilidades de afrontamiento, sus estructuras sociales, su auto-confianza y su sintomatología emocional y física.

\section{Sistema Penitenciario:}

El Sistema penitenciario, se modificó según la línea de pensamiento de la época. En 1838 en Pensylvania, U.S.A, se empieza a juzgar a niños como adultos, con el riesgo de ser abusados física y sexualmente por los adultos, y en 1960 vienen otras corrientes sociológicas, donde se cuidaban factores ambientales y externos que podían victimizar a los adolescentes.

En Costa Rica, se da el mismo proceso de desarrollo desde un régimen represor, que se basaba en la idea del castigo; hasta lo que se conoce como La Teoría de la Prevención del Delito (T.P.D), que busca la readaptación del privado de libertad basado en su derecho a la educación, al buen trato digno, a la no estigmatización (Ulate, 1997).

Pero debido a problemas de presupuesto, infraestructura y abandono político, estos ideales no se cumplen, y se dan situaciones 
de hacinamiento, tráfico y consumo de drogas, inseguridad y problemas de salubridad que lejos de readaptar al individuo lo hunden en un círculo vicioso de delincuencia y enfermedad mental.

\section{Improvisación Teatral:}

La improvisación teatral es una parte muy importante de la creación teatral para investigar la expresión corporal y vocal de intérprete $\mathrm{o}$ actor, donde su principal característica es la libertad de la imaginación, y su principal dificultad es la comunicación y sintonía grupal en el momento. Es un juego teatral que se sirve de los principios propios del teatro, agregándole sus propias reglas (Gravel, 1987).

Robert Gravel es el creador del match de improvisación y de la L.N.I (Liga Nacional de improvisación, de Canadá), la primera del mundo. Así se crea un juego donde el improvisador es entrenado para crear historias en el momento, jugando un rol de director, actor, y escritor al mismo tiempo. La regla principal de este juego es la no censura de la imaginación.

\section{¿Qué es un Match?}

Este juego fue creado en Canadá por Robert Gravel e Ivon Leduc en el año 1977, su estructura está basada en el jockey (deporte nacional de Canadá). Es un partido de improvisación entre dos equipos de improvisadores para ganar el punto otorgado por el público. El juego se realiza en una pista de Jockey, tiene una duración de 90 minutos, donde hay un árbitro que dirige el partido, dando los títulos de las improvisaciones y vigilando que no se hagan faltas, cada tres faltas es un punto al equipo contrario (Théatre Action, 1987).

La dinámica del partido es así:

$\checkmark$ Presentación de los equipos.

$\checkmark$ Himnos de ambos equipos.
Al pito del árbitro se empieza el partido: da el primer título su modalidad $\left(\mathrm{n}^{\circ}\right.$ de jugadores por equipo, estilo, duración, etc).

10 segundos para pensar y ponerse de acuerdo, cada equipo.

$\checkmark$ Inicia la improvisación.

$\checkmark \quad$ Al pito de árbitro concluye.

$\checkmark$ Se recuentan las faltas, que hubieron durante la improvisación, con cada capitán.

$\checkmark \quad$ El público vota.

$\checkmark$ La mayoría de votos es el punto para el equipo.

Así se realizan torneos y mundiales europeos, asiáticos y de habla hispana donde participan selecciones de cada país y cada continente.

\section{METODOLOGÍA}

Sujetos: participaron 21 varones adolescentes privados de libertad del Centro de Formación Zurquí, que no realizaban ningún tipo de actividad física dirigida. Algunos detalles de la muestra se describen a continuación:

Indiciados: 5 (estaban en espera de juicio y condena)

Edad promedio: $15,8 \pm 1,79$ años

Sentenciados: 16 (sujetos que ya tienen una condena)

Edad promedio: $16,125 \pm 0,81$ años

Instrumentos: POMS (Profile of Mood States; Mc Nair; Lorr y Droppleman, 1971). Este instrumento mide estados anímicos (tensión, depresión, fatiga, cólera y vigor). Se usó la adaptación española corta (Fuentes y cols., 1994, Fuentes y cols., 1995) de 15 ítems.

Procedimiento: se realizó una sesión de teatro de una hora, con el siguiente orden de actividades: 
* Explicación de las reglas del Match.

* División de equipos.

* Partido: Improvisación de títulos en fotografías corporales, primero propuestas por la investigadora, luego por el público (privados de libertad que no participaron y guardias de la prisión).

Se realizó una sesión de fútbol sala de una hora, con el siguiente orden de actividades:

* Explicación de las reglas.

* División de equipos.

* Partido: De tres equipos, en rotación por cada anotación.

No se realizó ningún tipo de calentamiento grupal en cada sesión, solo un estiramiento individual. Indiciados y sentenciados trabajaron por aparte, pues por reglas del Centro, no se les permite trabajar juntos. A los sentenciados se les aplicó primero la sesión de teatro y una semana después la de fútbol sala. A los indiciados, se les aplicó exactamente igual ambos tratamientos. El POMS se aplicaba inmediatamente antes y después de cada sesión.

Análisis estadístico: se realizó estadística descriptiva con promedios y desviaciones estándar. Como estadística inferencial, se aplicó ANOVA de 3 vías mixto (grupo $\mathrm{x}$ medición $\mathrm{x}$ tratamiento) y como post hoc el análisis de efectos simples y el análisis de interacciones simples, según fuese necesario. Todo se calculó con el programa SPSS (versión para Windows 8.0).

\section{RESULTADOS}

Tabla 1. Promedios y desviaciones estándar de los niveles de estado anímico de varones privados de libertad en función de grupo, tratamiento y medición

\begin{tabular}{|c|c|c|c|c|c|c|c|c|c|c|c|c|}
\hline & \multicolumn{4}{|c|}{ Indiciados $(n=5)$} & \multicolumn{4}{|c|}{ Sentenciados $(n=16)$} & \multicolumn{4}{|c|}{ Total $(n=21)$} \\
\hline & \multicolumn{2}{|c|}{ Teatro } & \multicolumn{2}{|c|}{ Fútbol sala } & \multicolumn{2}{|c|}{ Teatro } & \multicolumn{2}{|c|}{ Fútbol sala } & \multicolumn{2}{|c|}{ Teatro } & \multicolumn{2}{|c|}{ Fútbol sala } \\
\hline & Pre test & Post test & Pre test & Post test & Pre test & Post test & Pre test & Post test & Pre test & Post test & Pre test & Post test \\
\hline Tensión & $4,2 \pm 2,8$ & $1,4 \pm 1,7$ & $2,6 \pm 1,7$ & $4,4 \pm 2,9$ & $5,6 \pm 4,1$ & $3,2 \pm 2,9$ & $2,3 \pm 2,0$ & $4,0 \pm 2,5$ & $5,2 \pm 3,8$ & $2,8 \pm 2,8$ & $2,4 \pm 1,9$ & $4,0 \pm 2,6$ \\
\hline Fatiga & $3,0 \pm 3,7$ & $0,4 \pm 0,9$ & $1,8 \pm 1,8$ & $6,0 \pm 3,3$ & $4,0 \pm 3,9$ & $2,9 \pm 2,3$ & $2,9 \pm 3,08$ & $3,5 \pm 2,4$ & $3,7 \pm 3,8$ & $2,3 \pm 2,3$ & $2,7 \pm 2,8$ & $4,0 \pm 2,8$ \\
\hline Cólera & $4,0 \pm 3,9$ & $0,2 \pm 0,4$ & $0,8 \pm 1,8$ & $2,4 \pm 2,2$ & $4,4 \pm 3,8$ & $2,8 \pm 2,9$ & $2,9 \pm 3,0$ & $2,1 \pm 2,9$ & $4,3 \pm 3,7$ & $2,2 \pm 2,8$ & $2,4 \pm 2,9$ & $2,2 \pm 2,7$ \\
\hline Depresión & $4,4 \pm 2,2$ & $0,8 \pm 1,8$ & $0,8 \pm 1,8$ & $5,0 \pm 4,9$ & $4,75 \pm 4,4$ & $2,6 \pm 2,8$ & $3,4 \pm 3,5$ & $2,2 \pm 2,4$ & $4,7 \pm 3,9$ & $2,1 \pm 2,7$ & $2,8 \pm 3,4$ & $2,8 \pm 3,3$ \\
\hline Vigor & $10,4 \pm 2,2$ & $11,2 \pm 0,8$ & $9,4 \pm 3,9$ & $9,2 \pm 2,2$ & $7,2 \pm 4,3$ & $9,2 \pm 2,7$ & $8,0 \pm 3,6$ & $9,2 \pm 2,8$ & $7,9 \pm 4,1$ & $9,7 \pm 2,5$ & $8,3 \pm 3,6$ & $9,2 \pm 2,6$ \\
\hline
\end{tabular}

Tabla 2. Resumen de ANOVA aplicados a los estados de ánimo como variables dependientes en función de grupo, tratamiento y medición, en varones privados de libertad

\begin{tabular}{|c|c|c|c|c|c|c|c|c|c|c|c|c|c|c|}
\hline & \multicolumn{2}{|c|}{ Tratamiento } & \multicolumn{2}{|c|}{$\begin{array}{l}\text { Tratamiento } \\
\mathrm{X} \text { grupo }\end{array}$} & \multicolumn{2}{|c|}{ Medición } & \multicolumn{2}{|c|}{$\begin{array}{l}\text { Medición X } \\
\text { grupo }\end{array}$} & \multicolumn{2}{|c|}{$\begin{array}{l}\text { Tratamiento X } \\
\text { medición }\end{array}$} & \multicolumn{2}{|c|}{$\begin{array}{c}\text { Tratamiento X } \\
\text { medición X } \\
\text { grupo }\end{array}$} & \multicolumn{2}{|c|}{ Grupo } \\
\hline & $\mathrm{F}$ & Sig. & $\mathrm{F}$ & Sig. & $\mathrm{F}$ & Sig. & $\mathrm{F}$ & Sig. & $\mathrm{F}$ & Sig. & $\mathrm{F}^{\mathrm{s}}$ & Sig. & $\mathrm{F}$ & Sig. \\
\hline Tensión & 0,120 & 0,733 & 1,642 & 0,215 & 0,392 & 0,539 & 0,013 & 0,909 & 11,907 & $0,003 * *$ & 0,046 & 0,833 & 0,480 & 0,497 \\
\hline Fatiga & 0,196 & 0,663 & 0,714 & 0,409 & 1,862 & 0,188 & 2,940 & 0,103 & 7,483 & $0,013 *$ & 2,823 & 0,109 & 0,371 & 0,550 \\
\hline Cólera & 3,664 & 0,071 & 0,002 & 0,962 & 0,665 & 0,425 & 0,092 & 0,765 & 6,786 & $0,017 *$ & 3,701 & 0,070 & 1,800 & 0,196 \\
\hline Depresión & 1,828 & 0,192 & 3,750 & 0,068 & 0,096 & 0,760 & 0,400 & 0,534 & 10,214 & $0,005 * *$ & 6,099 & $0,023 *$ & 0,176 & 0,679 \\
\hline Vigor & 1,845 & 0,190 & 0,887 & 0,358 & 0,382 & 0,544 & 1,161 & 0,295 & 0,703 & 0,412 & 0,008 & 0,932 & 2,174 & 0,157 \\
\hline
\end{tabular}

$* \mathrm{p}<0,05 * * \mathrm{p}<0,01$ 
Gráfico 1. Interacción entre mediciones y tratamiento para la variable tensión medida en dos grupos de varones costarricenses privados de libertad

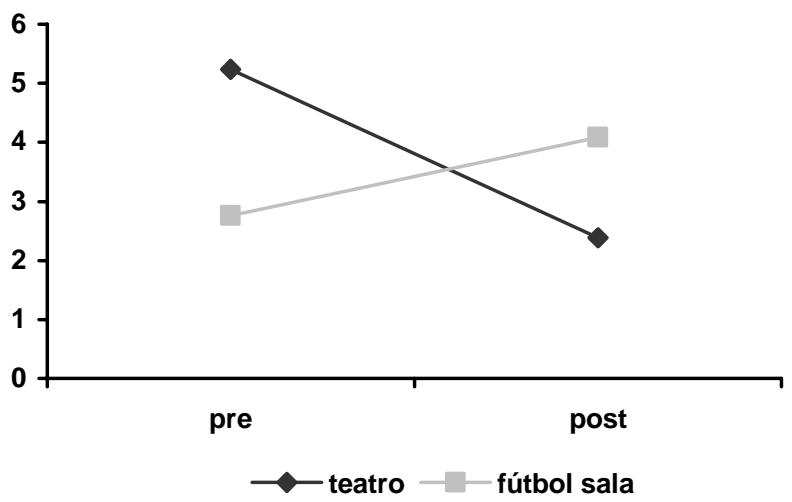

Gráfico 2. Interacción entre mediciones y tratamiento para la fatiga, medida en varones costarricenses privados de libertad

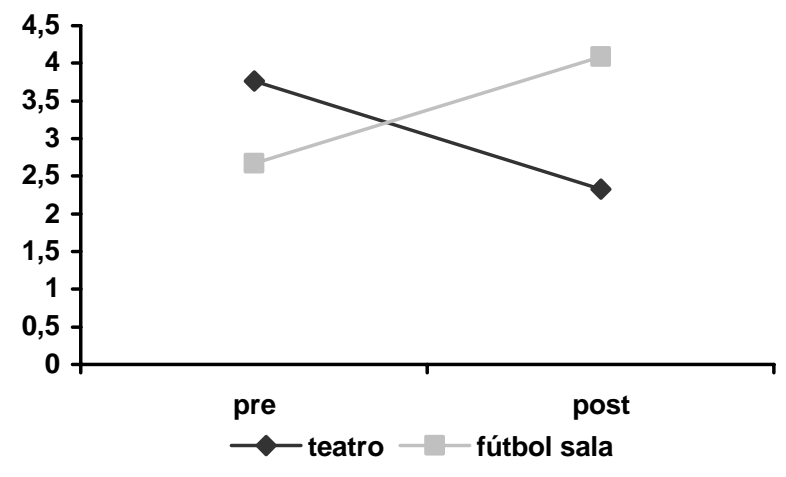

Gráfico 3. Interacción entre mediciones y tratamiento para cólera-ira medida en varones costarricenses privados de libertad

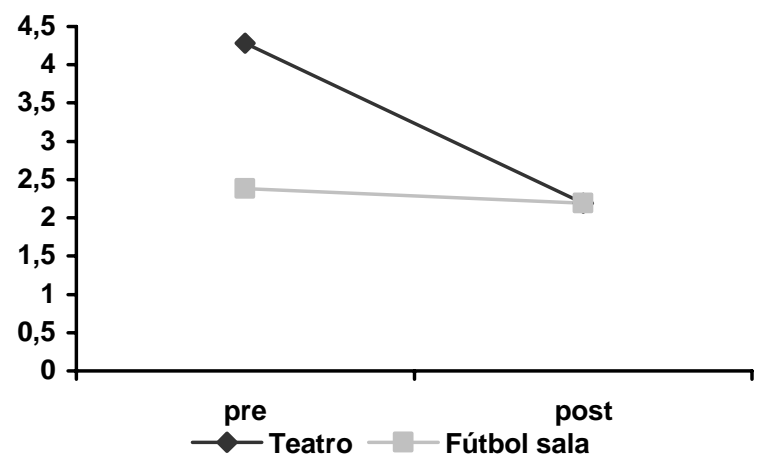

Gráfico 4. Interacción de mediciones, tratamiento y grupo para la depresión medida en varones costarricenses privados de libertad
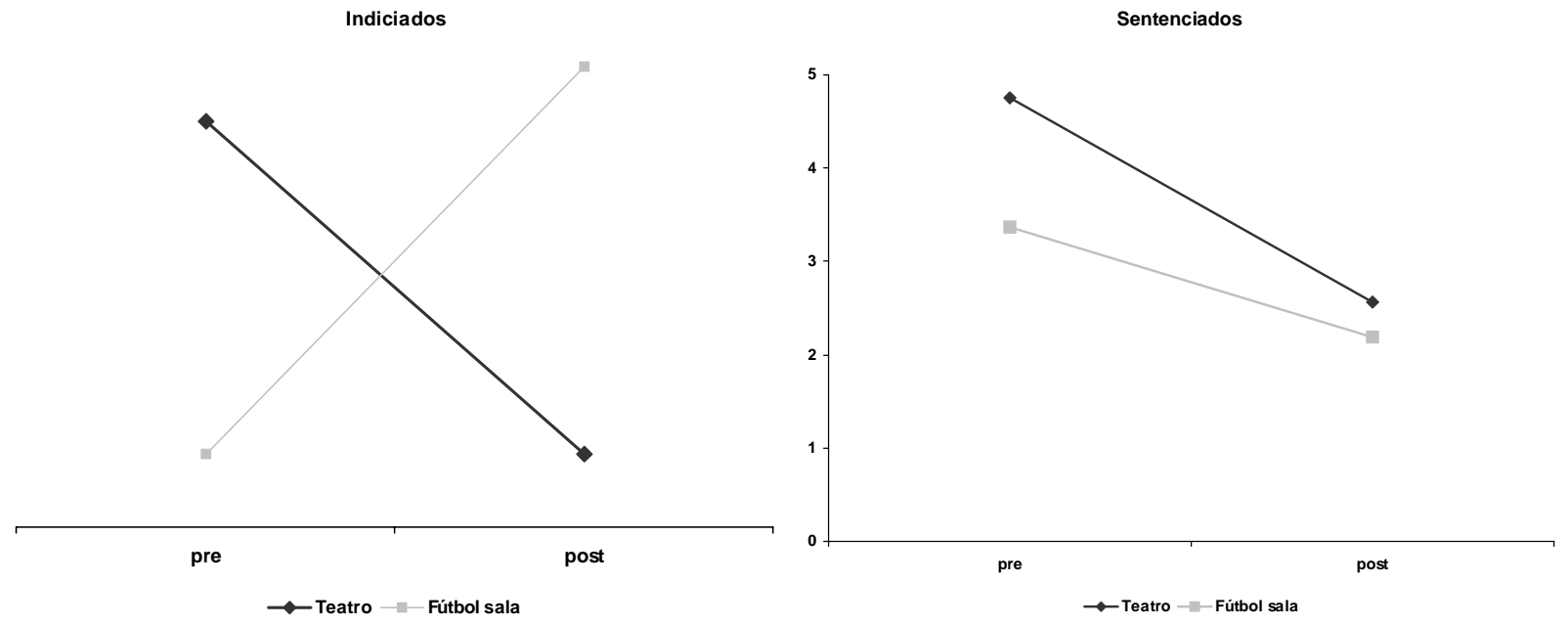
En la variable tensión, se encontró interacción significativa entre mediciones y tratamiento ( $\mathrm{F}: 11,907 ; \mathrm{p}<0,01)$. El análisis de efectos simples aplicados demostró diferencias significativas en el nivel de tensión al inicio de las sesiones de teatro y fútbol sala (al inicio de la sesión de teatro los sujetos experimentaron mayor tensión que al inicio de la sesión de fútbol); también se observó efecto agudo significativo en ambas sesiones, no obstante este efecto solo fue positivo para la sesión de teatro pues al final de la sesión de fútbol hubo un aumento significativo de la tensión. En fatiga, se encontró interacción significativa entre medición y tratamiento $(\mathrm{F}: 7,483 ; \mathrm{p}<0,05) . \mathrm{El}$ análisis de efectos simples mostró que solo existieron diferencias significativas en los niveles de fatiga al final de ambas sesiones (el nivel de fatiga fue mayor al final de la sesión de fútbol). En cólera-ira, se encontró interacción significativa entre medición y tratamiento $(\mathrm{F}: 6,786 ; \mathrm{p}<0,05)$. El análisis de efectos simples mostró que solo existió efecto agudo significativo en los niveles de cólera en la sesión de teatro (el nivel de cólera disminuyó significativamente al final de esta sesión). En depresión, se encontró interacción significativa entre medición, grupo y tratamiento (F: 6,099; p<0,05). El análisis de interacciones simples mostró que solo hubo efecto agudo significativo en los niveles de depresión en la sesión de teatro (El nivel de depresión disminuyó significativamente). En vigor no se encontró ningún efecto significativo. Es decir que ninguna de las condiciones experimentales logró afectarlo, significativamente.

\section{DISCUSIÓN}

Según los resultados encontrados, los efectos de teatro y más concretamente la técnica de improvisación, en el estado de ánimo, son positivos en sujetos privados de libertad. Según Allen y cols. (2002), si se reducen los patrones problemáticos en la adaptación de los adolescentes puede ocurrir una reducción de la incidencia de la delincuencia, lo cual permite suponer que este tipo de tratamientos que involucra el contacto social y la autoconfianza, pueden jugar un rol muy importante en la reducción de conductas delictivas.

Los resultados confirman la propuesta de Granello y Hanna de la instrucción artística, para buscar la libertad y la autonomía, factor decisivo en el desarrollo de la adaptación, la cual es estimulada en los dos tratamientos, pues cada sujeto cumple un rol decisivo para llevar adelante al equipo. Repasando cada una de las variables donde se encontró efecto, la variable tensión, estuvo alta al principio de la sesión de teatro, pero se dio una disminución significativa al final de la sesión, al contrario del tratamiento deportivo; se supone que por ejemplo el miedo al ridículo (factor que juega un rol muy importante, en el aprendizaje teatral), pudo influir permitiendo que los sujetos conforme avanzaba la sesión se relajaran y les permitiera jugar más, al contrario del fútbol o fútbol sala, deportes muy conocidos por la mayoría de los sujetos y manipulados para demostrar el poderío, característica importante en esta población, (Kunzi,1999), lo cual pudo haber llevado a un aumento muy circunstancial de la variable. Además la fatiga, otra variable importante en el desarrollo de la investigación, se vio aumentada significativamente en el fútbol sala, por razones obvias de intensidad del ejercicio. Una situación importante de mencionar, es que en uno de los partidos de fútbol se dieron muchos roces por faltas controversiales y cambios de algunos sujetos de un equipo a otro e impedimento entre ellos para pertenecer a los equipos, lo que pudo influir en el aumento de depresión al final de la sesión. El fútbol como deporte más popular, permite ser más manipulado para pelear y manifestar conductas violentas. En la improvisación teatral, en cambio, al tomarse menos en serio como competencia, permitió que esas características del juego-deporte no salieran a flote. 
La cólera y la depresión son rasgos característicos de este tipo de población (Kunzi, 1999; Granello y Hanna, 2003; Forrest y cols., 2000), y fueron trabajados en la improvisación, por su efecto de catársis y por el estímulo de la imaginación que provoca una sensación de poder y autoconfianza, de acuerdo con Johnstone (1979). Su disminución aguda permite deducir que la improvisación introduce un efecto de relajación progresiva, al verlo como un juego infantil.

Se respalda a Sussman y cols. (2000), Forrest y cols. (2000), en cuanto a la necesidad de este tipo de programas de rehabilitación para esta población; la improvisación se vuelve un descubrimiento importante como actividad recreativa, para el estímulo de la imaginación, la catársis, y la relajación de este tipo de adolescentes

Las características de estos adolescentes son importantes variables a tomar en cuenta, para poder trabajar con ellos, ya que manejan mucha ansiedad, depresión, síndrome de abstinencia, percepción de soledad, optimismo exagerado, e inseguridad por su situación, por lo que demandan diferentes estrategias para la motivación de participar. Este estudio refuerza estudios nacionales, anteriores, que han demostrado efectos positivos en el estado de ánimo, y otras variables psicológicas, por medio de la actividad física recreativa o competitiva (Ureña, Mora, Godínez y Rodríguez, 2004; Calderón, Delgado y Oviedo, 2005). No obstante, en los resultados se evidencia que la actividad deportiva no tendió a beneficiar el estado de ánimo de los privados de libertad.

El sistema penitenciario no le da la importancia requerida a los programas de actividad física recreativa o deportiva, recortando presupuesto y personal, y manteniendo a los adolescentes con el nivel de actividad física por debajo del "mínimo recomendado" por el Manual de la buena práctica penitenciaria, regla: 21 . Por lo tanto, se concuerda con Vargas $\left(1995_{\mathrm{a}}\right.$ y $\left.\mathrm{b}\right)$, en cuanto a la necesidad de darle importancia a la participación voluntaria de los sujetos en este tipo de programas, para así mejorar su calidad de vida.

En síntesis, esta línea de estudio es prometedora y debería profundizarse más. Así mismo, es importante la investigación del teatro y la expresión corporal, pues sus efectos terapéuticos, pueden ser introducidos como tratamientos de bajo costo. Además, es necesario darle mayor importancia a la población de adolescentes privados de libertad, dadas sus características especiales.

\section{REFERENCIAS}

Allen, P. J., Marsh, P., McFarland, C., McElhaney, B. K., \& Land, D. (2002). Attachment and Autonomy as predictors of Development of Social Skills Delinquency during Midadolescence. Journal of Consulting and Clinical Psychology, 70(1): 6-66.

Berger, B.G. y Motl, R.W. (2000). Exercise and Mood: A selective Review and Synthesis of Research Employing the Profile of Mood States. Journal of Applied Sport Psychology, 12: 6992

Biddle, S. (1993). Children, Exercise and Mental Health. International Journal of Sport Psychology, 24: 200-216.

Calderón, E, A.; Delgado, M, M. y Oviedo, S.; M. (2005). Efecto de un Programa Físico Recreativo y otro de Actividades Deportivas Competitivas sobre la auto-estima, y los estados de ánimo en Privados de Libertad del Centro de Atención Institucional Dr. Gerardo Rodríguez Echeverría. Tesis para optar por el grado de licenciatura en ciencias del deporte con énfasis en salud. Escuela Ciencias del Deporte. Facultad de Ciencias de la Salud. Universidad Nacional.

Dishman, K. R. \& Jackson, M.E. (2000). Exercise, Fitness, and Stress. International Journal of Sport Psychology, 31: 175-203.

Dubbert, M.P., (2002). Physical Activity and Exercise: Recent Advances and Current Challenges. Journal of Consulting and Clinical Psychology, 70(3): 526-536.

Forrest, B, C., Tambor, E., Riley, W, A., Ensminger, E, M., \& Starfield, B. (2000). The Health Profile of Incacerated Male Youths. Pediatrics. 105(1): 286-291.

Fuentes, I.; Balaguer, I.; Meliá, J. y García-Merita, M. (1994). Formas paralelas de la adaptación valenciana del perfil de ánimo (POMS). Comunicación presentada en el IV Congreso 
de evaluación psicológica. Santiago de Compostela. 21-24 setiembre.

Fuentes, I.; Balaguer, I.; Meliá, J. y García-Merita, M. (1995). Forma abreviada del perfil de ánimo (POMS). Comunicación presentada en el V Congreso de psicología de la actividad física y el deporte. Valencia. 22-24 marzo.

Granello, F, P., \& Hanna, J, F. (2003). Incarcelet and Court-Involved Adolescents: Counseling and At-Risk Population. Journal of Counseling \& Development. 81:11-18.

Gravel, R. (1987). Impro, reflexiones y análisis. Canadá: Edición Leméac.

Johnstone, K. (1979). Improvisación y el teatro. Chile: Editorial Cuatro Vientos.

Kirkcaldy, D. B. \& Shephard, J. R. (1990). Therapeutic Implications of Exercise. International Journal of Sport Psychology, 21: 165-184.

Kunzi, V.I. (1999). Estilo Atributivo, Percepción de Soledad y Estrategias de Afrontamiento en Adolescentes Bajo Tutela Judicial. Revista Interdisciplinaria, 2:169-186.

Martinsen, W. E. (1993). Therapeutic Implications of Exercise for Clinically Anxious and Depressed Patients. International Journal of Sport Psychology, 24:185-199.

O’Neal, A. H., Dunn, 1. A., \& Martinsen, W. E. (2000). Depression and Exercise. International Journal of Sports Psychology, 31: 110-135.

Smith, J. C. y Crabbe, B. J. (2000). Emotion and Exercise. International Journal of Sport Phychology, 31: 156-174.

Sussman, P, M., Jones, E, S., Wilson, W, T., \& Kann, L.(2002). The Youth Risk Behaviors Surveillance System: Updating Policy and Program Applications. Journal of School Health. 72(1): 13-17.

Tréatre Action. (1987). Rompan el Hielo. Ontario, Canadá, Publication.

Tucker, A. L. (1990). Physical Fitness and Psychological Distress. International Journal of Sport Psychology, 21: 185-201.

Ulate, F. (1997). El Régimen disciplinario aplicado a los privados y privadas de libertad en la institución carcelaria: Una especial referencia al centro femenino de atención institucional El Buen Pastor. Tesis de grado para optar al grado de licenciatura en derecho. Facultad de Derecho. Universidad de Costa Rica.

Ureña, A, R., Mora, M,J., Godínez, S, F. y Rodríguez, V, W. (2004). Desarrollo e implementación de un programa de actividad física para la reducción de la ansiedad en varones adultos, mayores de cincuenta años, privados de libertad. Tesis para optar por el grado de licenciatura en ciencias del deporte con énfasis en salud. Escuela Ciencias del Deporte. Facultad de Ciencias de la Salud. Universidad Nacional.
Vargas, O. (1995a). Efecto del Ejercicio de la Imagen Corporal de Drogadictos. Revista Educación de la Universidad de Costa Rica. 19(2): 5158.

Vargas, O. (1995 $)$. Efecto de un Programa de Actividades Físicas en constructos de la personalidad de Drogadictos. Tesis sometida a la consideración de la comisión del Programa de Estudios de Posgrado en Ciencias de la Educación para optar por el grado de Magister Scientiae. Universidad de Costa Rica. 